\title{
Leitlinienbasierter Clinical Decision Support - Anforderungen an evidenzbasierte Entscheidungsunterstützungssysteme
}

Philipp Börm

\section{Einführung}

Seit der Einführung von Computern sind auch Computersysteme zur Unterstützung klinischer Entscheidungen entwickelt und in der klinischen Medizin getestet worden. Ein Clinical-Decision-Support-System (CDS-System) ist eine Computersoftware, die Ärzte bei Entscheidungsaufgaben unterstützt, z. B. bei der Ermittlung einer Diagnose auf der Grundlage von Patientendaten. Der Hauptzweck moderner CDS-Systeme besteht darin, Kliniker am Ort der Versorgung zu unterstützen [1]. Computergestützte CDS-Systeme könnten im Gesundheitswesen transformativ sein. Doch trotz zahlreicher enthusiastischer Versuche über mehr als 4 Jahrzehnte und überzeugender Demonstrationen der Nutzen ist die Einführung von CDS-Systemen nur im Schneckentempo vorangekommen [2].

Klinische Leitlinien sind systematisch entwickelte Feststellungen (engl. statements), die Ärzte, Angehörige anderer Gesundheitsberufe und Patienten bei ihren Entscheidungen über die angemessene Gesundheitsversorgung unter spezifischen klinischen Umständen unterstützen sollen [3]. Klinische Leitlinien werden vom US Institute of Medicine (IOM) auch definiert als Empfehlungen zur Optimierung der Patientenversorgung, die auf einer systematischen Überprüfung der Evidenz und einer Bewertung der möglichen Nutzen und Schäden (engl. benefits, harms) beruhen [4]. Klinische Leitlinien werden normalerweise von medizinischen Experten oder Gremien entwickelt, die von Fachgesellschaften einberufen werden. Die Experten überprüfen relevante Studien zu Nutzen und Risiken ärztlicher Anwendungen und führen Metaanalysen durch, indem sie die Ergebnisse verschiedener Studien vergleichen und kombinieren [2]. Die Evidenzen werden in Leitlinien zusammengefasst und Ärzten zur Unterstützung ihrer klinischen Entscheidungen zugänglich gemacht.

\section{Nutzen und Risiken von Leitlinien und von Clinical Decision Support}

Klinische Leitlinien sollen Fehler und Unterschiede in der Versorgung sowie Verschwendung von Ressourcen reduzieren und gleichzeitig bewährte Verfahren und Verantwortlichkeiten in der Medizin unterstützen [2]. Wenn
Leitlinien befolgt werden, verbessern sich die klinischen Ergebnisse [5]. Zahlreiche Studien haben jedoch gezeigt, dass Kliniker Leitlinien häufig nicht befolgen. Dies tritt in allen Fachgebieten und in allen Ländern auf [5]. Als Gründe für die Nichteinhaltung von Leitlinien werden u. a. genannt, dass Leitlinien zu komplex seien, zu viele, sogar widersprüchliche Regeln enthielten, dass Leitlinien nicht aktuell seien, Ärzte andere persönliche Ansichten verträten oder sich selbst überschätzten. Gründe könnten auch eine falsche Risikobewertung, Zeitdruck oder eine Überflutung an Information sein [5].

In Studien konnte gezeigt werden, dass die Anwendung von CDS-Systemen die Leitlinienadhärenz erhöht [6]. Insbesondere wenn CDS-Systeme am Ort der Versorgung (engl. point of care) angewendet und gut in Workflows integriert werden, erhöhen sie die Leitlinienadhärenz, verbessern die Angemessenheit der Überwachung und Behandlung von Krankheiten und sorgen für eine verbesserte Anwendung von Medikamenten [7]. Die Implementierung von CDS-Systemen hat das Potenzial, Effizienz und Präzision der Versorgung zu verbessern, insbesondere in Disziplinen, die komplexe Entscheidungen erfordern. Clinical Decision Support kann zu Kostenersparnissen im Gesundheitssystem führen [8], durch klinische Interventionen [9], durch die Verkürzung der stationären Verweildauer, durch den Vorschlag kostengünstigerer Medikamentenalternativen [10] oder durch die Reduzierung von Doppeluntersuchungen [11]. Die Vorteile umfassen neben einer Reduzierung unerwünschter Arzneimittelwirkungen [12] auch verringerte Mortalitätsrisiken [13]. CDS-Systeme, die auf Sicherheit abzielen, unterstützen durch automatisierte Warnungen die Vermeidung von Arzneimittelereignissen, z. B. Verschreibungs- und Dosierungsfehler oder Anwendungsfehler bei Kontraindikationen [14]. Es wurde auch gezeigt, dass CDS das 10Jahres-CV-Risiko (CV: kardiovaskulär) bei Erwachsenen signifikant verbessern kann [15].

Potenzielle Risiken von CDS-Systemen umfassen eine abnehmende Aufmerksamkeit des Arztes gegenüber Warnmeldungen (engl. alarm fatigue), verlängerte Interaktionszeiten mit der Technologie, die Generierung von Umgehungslösungen (engl. workarounds) durch den Arzt und neue Fehler bei der Verschreibung von Medikamenten [7]. 
Ziele der angestrebten Innovationen durch CDS-Systeme sind die Verbesserung von Qualität (Leitlinienadhärenz, bessere Outcomes), Effizienz (Kosten), Sicherheit (Patientenschäden) und Transparenz (warum welche Intervention) in der Versorgung von Patienten. Die Anwendung leitlinienbasierter CDS-Systeme könnte die Befolgung von Leitlinien möglicherweise weiter steigern und Versorgungsergebnisse zusätzlich verbessern.

\section{Stand des Wissens}

\section{Einteilung von Clinical-Decision- Support-Systemen}

Generell können CDS-Systeme in wissensbasierte und nicht wissensbasierte CDS-Systeme unterteilt werden. Bei wissensbasierten CDS-Systemen kann zwischen Bayes'schen Netzwerken, kausalen probabilistischen Netzwerken und regelbasierten Systemen unterschieden werden. Bayes'sche Netzwerke sind die grafische Darstellung von Variablen und ihren Wahrscheinlichkeitsbeziehungen und basieren auf bedingten Wahrscheinlichkeiten von Ereignissen bei Auftreten anderer Ereignisse. Die Regeln dieser Netzwerke ermöglichen die Berechnung der Wahrscheinlichkeit von Ereignissen bei Vorliegen anderer Ereignisse. Bayes'sche Netzwerke werden z. B. verwendet, um die Wahrscheinlichkeit des Vorhandenseins möglicher Krankheiten aufgrund von Symptomen zu berechnen.

Kausale probabilistische Netzwerke basieren auf Ursache und Wirkung. Es wird dabei ein Pfad von den Symptomen bis zur Klassifizierung einer Krankheit verfolgt und anhand von Wahrscheinlichkeiten bestimmt, welcher Pfad im konkreten Fall am besten passt. Diese Methode wird angewendet, um z. B. das Fortschreiten einer Krankheit über die Zeit und die Wechselwirkung zwischen Krankheiten zu modellieren.

Regelbasierte Systeme beabsichtigen, das Wissen von Experten in Regeln auszudrücken und die Regeln miteinander zu verketten, um zu Schlussfolgerungen zu gelangen. Regelbasierte Systeme geben die Logik wieder, die in einem ärztlichen Entscheidungsprozess verwendet wird. Die Regeln und ihre Verkettungen werden in einer Wissensbasis (Regeldatenbank; engl. knowlegdebase) festgehalten.

Nicht wissensbasierte CDS-Systeme verwenden maschinelles Lernen (ML), eine Form der künstlichen Intelligenz. Maschinelles Lernen ermöglicht es Computern, aus früheren Ereignissen zu lernen und/oder Muster in klinischen Daten zu finden. Dadurch entfällt zwar der Prozess des Verfassens von Regeln durch Experten. Aber MLbasierte Systeme können bisher nicht die Gründe für ihre Schlussfolgerungen erklären. Sie sind sog. „Black Boxes“, in denen für Menschen bisher keine aussagekräftigen In- formationen über ihre Funktionsweise erkennbar werden. Insgesamt werden CDS-Systeme ohne Wissensbasis noch selten verwendet.

Da in Leitlinienaussagen Regeln wiedergegeben werden, unter welchen klinischen Bedingungen nämlich welche Anwendungen durchzuführen sind, sind leitlinienbasierte CDS-Systeme den regelbasierten Systemen zuzuordnen.

\section{Klinisches Problem, Problemliste}

Lawrence Weed hat 1968 die Logik der problemorientierten Krankenakte eingeführt (engl. problem-oriented health record; Weed, 1968 [16]). Jede Krankenakte soll demnach eine Liste aller Probleme des Patienten enthalten, einschl. der bereits bestehenden Diagnosen (engl. established diagnoses) und der ungeklärten Befunde (engl. unexplained findings), also der Befunde, die nicht mit den bereits festgestellten Diagnosen erklärt werden können [17]. Die Problemliste ist nicht statisch, sondern ein dynamisches Inhaltsverzeichnis der Patientenakte, das ständig aktualisiert wird [16]. Während des Behandlungsprozesses sollten die ungeklärten Befunde der Problemliste einer Diagnose zugeordnet werden [18]. In der Problemliste kann u.a. zwischen akuten und chronischen und zwischen aktiven und inaktiven Problemen unterschieden werden. Aktive Probleme erfordern die aktuelle Aufmerksamkeit des Arztes. Gelöste Probleme können das Management aber auch beeinträchtigen und sollten ebenfalls berücksichtigt werden. Die problemorientierte Krankenakte ist so aufgebaut, dass einzelne Anteile, z. B. Untersuchungsbefunde und Interventionen, den klinischen Problemen des Patienten (der Problemliste) klar zugeordnet werden können.

Die Problemliste stellt ein sehr wesentliches, für die Anwendung einer computerbasierten klinischen Entscheidungsunterstützung relevantes klinisches Ordnungsprinzip dar. Für jedes Patientenproblem könnte es eine vordefinierte Problemlösungsstrategie in Form eines spezifischen problembasierten CDS-Tools geben. Dieses CDS-Tool wäre eine Zusammenstellung der bei diesem Problem zu berücksichtigenden Entscheidungsregeln. Problemlisten könnten somit zur Kontextualisierung von CDS-Tools angewendet werden.

\section{Unterschied zwischen narrativen und maschinenlesbaren Inhalten}

Traditionell werden Informationen, Erfahrungen und Kenntnisse, so auch klinisches Leitlinienwissen, von Inhaltsentwicklern in „narrativer“ Form (Textform), z. B. in Leitlinientexten, Endbenutzern zur Verfügung gestellt, d. h. in Sequenzen von geschriebenen oder gesprochenen Worten. Narrative Inhalte müssen von Menschen gelesen oder angehört, verstanden und dann verarbeitet werden. Narrative Inhalte sind per se nicht wirklich maschinenlesbar. Computer können narrative Informationen nicht direkt verarbeiten und ausführen. 
- Tab. 1 Leitlinien-Repräsentationsmodell (Boxwala-Modell), adaptiert nach: Boxwala et al., 2011 [18]; Centers for Disease Control and Prevention, $2018[20]$.

\begin{tabular}{|l|l|l|l|}
\hline Level & Typ & Beschreibung & Formate \\
\hline L1 & narrativer Text & narrativer Text zu einer bestimmten Krankheit & $\begin{array}{l}\text { veröffentlichte Leitlinie, von Experten begutach- } \\
\text { teter Artikel, andere unstrukturierte Formate }\end{array}$ \\
\hline L2 & semistrukturiert & $\begin{array}{l}\text { Kernkonzept ist Empfehlung: modelliert als Entscheidung } \\
\text { über Interventionen in bestimmten klinischen Szenarien }\end{array}$ & $\begin{array}{l}\text { Leitlinienaussage, Flussdiagramm, Entschei- } \\
\text { dungsbaum, andere ähnliche Formate }\end{array}$ \\
\hline L3 & strukturiert & $\begin{array}{l}\text { spezifizierte Empfehlung mit ausreichender Struktur, um sie } \\
\text { präzise und ausführbar zu machen; modelliert als Entschei- } \\
\text { dung in bestimmten klinischen Szenarien }\end{array}$ & $\begin{array}{l}\text { standardkonforme Spezifikationen, Codierungs- } \\
\text { logik mit Datenmodellen, Terminologien, vor- } \\
\text { bereitet für Implementierung }\end{array}$ \\
\hline L4 & CDS, implementiert und verwendet in einer lokalen & CDS-Tool \\
\hline KIS: Krankenhausinformationssystem & Ausführungsumgebung (z. B. KIS) & \\
\hline
\end{tabular}

CDS-Systeme sind dagegen maschinenlesbar, d. h. sie bilden medizinische Information in Form von Software ab. Sie können bei entsprechender Eingabe von Inputdaten auf Computern ausgeführt werden und einen Output anzeigen. Interaktive Software akzeptiert dabei die Eingaben von Menschen. Interaktive CDS-Softwareinstrumente stellen eine Echtzeitinteraktion zwischen Computer und Mensch dar.

\section{Leitlinien-Repräsentationsmodell (Boxwala-Modell)}

2011 schlugen Boxwala et al. [19] ein allgemeines mehrschichtiges Repräsentationsmodell für Leitlinienwissen vor, das die Übersetzung klinischen Wissens in digitale Formen beschreiben sollte ( $\bullet$ Tab. 1).

Die Übersetzung klinischen Wissens in digitale Formen entsprechend dem Boxwala-Modell ermöglicht neben einer Maschinenlesbarkeit der Inhalte auch eine Transportierbarkeit (engl. portability, deployability) dieses digitalen Wissens auf unterschiedliche klinische Standorte. Das Boxwala-Modell ist sehr hilfreich bei der Formulierung von Anforderungen an ein wirklich digitales leitlinienbasiertes CDS-System.

\section{Mathematische Konzepte}

Mathematische Konzepte sind der Schlüssel, um Medizin und medizinische Daten in Softwaresystemen zu organisieren und klinische Probleme und Lösungen maschinenlesbar, maschinenverständlich und maschinenverarbeitbar und somit auch für den Menschen nutzbar zu machen. Für das Verständnis klinischer Entscheidungsunterstützungssysteme (CDSS) ist die Kenntnis der Grundprinzipien von Logik und Wahrscheinlichkeit von wesentlicher Bedeutung [1]. CDS-Systeme basieren auf der Anwendung der mathematischen Konzepte der Mengenlehre, der Boole'schen Algebra, der Wahrscheinlichkeitstheorie, der Fuzzy-Logik und der Entscheidungstheorie. Außerdem ist für klinische Entscheidungen die Anwendung einer Risikomanagementtheorie unerlässlich.
Die Mengenlehre (engl. Set Theory) ist ein Teilbereich der mathematischen Logik. Die Menge (engl. Set) ist ein genau definierter Verbund eindeutiger Objekte oder Elemente (z.B. die Menge von Polygonen). Mengen werden als grundlegendes Konzept der Mathematik auch verwendet, um logische Operationen so zu formalisieren, dass Maschinen (Computer) sie verstehen können. Die Boole'sche Algebra ist ein Formalismus zur Beschreibung logischer Beziehungen, so wie die Algebra numerische Beziehungen beschreibt. Boole'sche Operatoren können in der Medizin angewendet werden, um z.B. logische Verknüpfungen zwischen klinischen Ausgangssituationen und anzuwendenden ärztlichen Eingriffen aufzuzeigen. Computergestützte Entscheidungssysteme können eine numerische Darstellung von Wahrscheinlichkeiten (d.h. Wahrscheinlichkeitstheorie) benutzen, um Aussagen zu Schlussfolgerungen zu kombinieren. Entscheidungstheorie wird in der Medizin angewendet bei klinischen Entscheidungsregeln, bei denen Verzweigungslogik in Kombination mit Daten verwendet wird, um Schlussfolgerungen zu ziehen hinsichtlich Diagnose, Behandlung und anderer wichtiger klinischer Ziele. Fuzzy-Logik wird verwendet, um Entscheidungsprobleme zu lösen, bei denen Unsicherheit eine Rolle spielt. Beim Risikomanagement geht es um die Schweregrade und Eintrittswahrscheinlichkeiten von Schäden, aber auch von Nutzen klinischer Eingriffe.

\section{PICO-Modell}

Das PICO-Modell dient ebenfalls einer formalisierten Darstellung klinischer Entscheidungsregeln. Ursprünglich entwickelt als Grundsyntax in der evidenzbasierten Medizin, um eine Frage für die Literatursuche zu bilden und dann geeignete Ressourcen über klinische Studien und relevante Evidenz zu identifizieren, steht PICO für: $\mathrm{P}=\mathrm{Po}$ pulation, Patientenmerkmale, I= Intervention, $C=$ Vergleich (engl. comparison), $\mathrm{O}=$ Ergebnis (engl. outcome). PICO beschreibt also die zu beobachtende oder zu behandelnde Patientenpopulation anhand von Patientencharakteristika $\mathrm{P}$, die Intervention I, alternative Interventio- 
nen $\mathrm{C}$ und die zu erwartenden Ergebnisse O. Das PICOModell verwendet bei der Formulierung der Frage, d.h. bei der Verknüpfung der Ps, Is, Cs und Os ganz einfache mathematische Prinzipien, wie eben die Mengenlehre, Aussagenlogik, Boole'sche Operatoren, Wahrscheinlichkeiten, und übertragen auf die Anwendung im klinischen Alltag auch entscheidungs- und risikomanagementtheoretische Prinzipien. Das PICO-Modell ist ein weiterer Baustein, der bei der digitalen Transformation von klinischem Wissen in digitale Artefakte angewendet werden könnte.

\section{Terminologien, Vokabulare, Datenmodelle}

Um die Computerlesbarkeit und Interoperabilität von Leitlinien und Leitlinien-Statements weiter zu erhöhen, können inzwischen entwickelte, standardisierte Terminologien und Datenmodelle zur Kennzeichnung und Identifizierung angewendet werden. Hier eine Auswahl:

- ICD10/11: medizinische Klassifizierung der WHO, mit Codes u.a. für Krankheiten, Befunde, Symptome, soziale Umstände und Ursachen von Krankheiten

- SNOMED CT (engl. Systematized Nomenclature of Medicine Clinical Terms): standardisiertes, mehrsprachiges Vokabular für den elektronischen Austausch klinischer Gesundheitsinformationen

- LOINC: Sprache (Begriffe, Codes) zur Beschreibung von Gesundheitsmaßen, Beobachtungen und Dokumenten

- ATC/DDD (engl. anatomical therapeutic chemical classification system): Klassifikation von Arzneimitteln

- COS: Modell von Cochrane und der COMET-Initiative (engl. Core Outcome Measures in Effectiveness Trials) zur Klassifikation von Core Outcome Sets (COS), also der Ergebnisse klinischer Aktivitäten (s. auch Davey Classification; Davey, 2011 [21])

- OMOP CDM (engl. Common Data Model der Observational Medical Outcomes Partnership): international anerkanntes US-amerikanisches Datenmodell, mit dem Daten aus verschiedenen Datenbanken in ein gemeinsames Format und eine gemeinsame Darstellung (Terminologien, Vokabulare, Codierungsschemata) umgewandelt werden, zur systematischen Analyse unter Verwendung einer Standardbibliothek

- OHDSI-Programm (engl. Observational Health Data Sciences and Informatics, ausgesprochen „Odyssey“): interdisziplinäre Zusammenarbeit, um den Wert von Gesundheitsdaten durch umfangreiche Analysen mithilfe des OMOP-CDM-Datenmodells herauszustellen

Der Austausch von Patientendaten zwischen verschiedenen digitalen Systemen im Gesundheitswesen kann nur funktionieren, wenn diese Terminologien und Datenmodelle angewendet werden. Patientendaten, die mithilfe ausführbarer CDS-Tools gesammelt werden und die mit diesen Terminologien gekennzeichnet sind, sind maschinenlesbar, maschinenverarbeitbar und automatisiert analysierbar. Sie sind somit auch die ideale Grundlage für ML-basierte Anwendungen.

\section{Anforderungen an leitlinienbasierten Clinical Decision Support}

\section{Definition des leitlinienbasierten CDS}

Unter einem leitlinien-, d.h. regelbasierten klinischen Entscheidungsunterstützungssystem (CDSS) soll ein Softwaresystem verstanden werden, das automatisierte Argumentation auf digitalisierte Patientendaten gemäß bereitgestellter klinischer Regeln anwendet und Benutzern (Ärzten) digitalisierte Leitlinienempfehlungen für die Diagnose, Prävention, Überwachung, Behandlung oder Linderung von Krankheiten anzeigt. „Klinische Entscheidung“ (engl. decision) soll die tatsächliche Auswahl der dann auszuführenden Behandlung (Aktivität) bedeuten [2]. „Unterstützung“ soll dagegen die Hilfestellung bei der Entscheidungsfindung bedeuten [2], d. h. Benutzern sollen die passenden Empfehlungen lediglich aufgezeigt werden. Es gibt bei CDS-Systemen immer einen Vermittler (Empfänger, Benutzer) in der Schleife zwischen der Empfehlung einer Entscheidung, der Entscheidung und der tatsächlichen Aktivität. Die klinische Entscheidungsunterstützung ist daher kein „geschlossener“ Prozess [2].

\section{Struktur klinischer Entscheidungsregeln}

Ein Arzt trifft seine klinischen Entscheidungen auf der Grundlage klinischer Entscheidungsregeln, die Teil seines persönlichen medizinischen Wissens sind. Ein CDS-System sollte diese Entscheidungsprozesse unterstützen, indem Empfehlungen auf der Grundlage klinischer Entscheidungsregeln (oder Produktionsregeln) präsentiert werden, die zuvor festgelegt und in der CDSS-Wissensbasis gespeichert werden. Eine klinische Entscheidungsregel sollte die Merkmale der Situation dem Verhalten zuordnen, das durchgeführt werden soll, oder den Schlussfolgerungen, die in dieser Situation gezogen werden sollen.

Das herkömmliche Format für eine Entscheidungsregel ist die IF-THEN-Aussage: WENN <Bedingung> DANN $<$ Aktion>. Dabei steht < Bedingung > für eine logische Aussage, die, falls zutreffend, zur Durchführung der <Aktion> führen soll. Die Bedingung kann einen einfachen Einzelabgleich mit Daten bedeuten oder es kann sich um eine komplexe Aussage der Boole'schen Logik handeln, bei der Assoziationen, Konjunktionen, Disjunktionen und Negationen in Bezug auf Daten verwendet werden. Die $<$ Aktion> einer Produktionsregel kann eine Anweisung zum Generieren einer Nachricht sein, normalerweise einer Empfehlung, oder eine Schlussfolgerung, die ggf. weitere Datenelemente abfragt und dem CDSS verfügbar macht. Darüber hinaus können Bedingung und Aktion einer Entscheidungsregel beliebig komplex sein und aus mehreren Bedingungen und mehreren Aktionen bestehen. Eine regelbasierte Wissensbasis besteht aus einer Sammlung dieser Entscheidungsregeln und damit auch aller Datenelemente, die den Zustand eines Patienten beschreiben. Leitlinienbasierte Entscheidungsregeln sind 
die Übersetzung ausführbarer Leitlinienaussagen (engl. executable key action statement, [22]).

\section{Hauptkomponenten von CDS-Systemen}

Die meisten regelbasierten CDS-Systeme bestehen aus den 5 Komponenten Wissensbasis, Inferenzmaschine, Erklärungskomponente, Benutzeroberfläche und Patientendatenbank. Die Wissensdatenbank ist eine Bibliothek klinischer Entscheidungsregeln (IF-THEN-Regeln oder Bedingungs-Aktions-Anweisungen) und Zusammenstellungen dieser Entscheidungsregeln (z. B. in problembasierten CDS-Tools). Die IF-Bedingungen der Entscheidungsregeln sind klar definierte strukturierte medizinische Eingabeelemente (Patientendaten; Begriffe, Modifikatoren), die THEN-Maßnahmen sind digitalisierte Ausgabeempfehlungen. Eine Inferenzmaschine (engl. inference engine; Regelinterpreter, Schlussfolgerungsmaschine) kombiniert die Regeln aus der Wissensbasis mit den eingegebenen Patientendaten und demonstriert eine oder mehrere digitalisierte Empfehlungen (Ausgaben). Die automatisierte Argumentation erfolgt im Hintergrund. Eine Erklärungskomponente (oder Begründer) hilft dem Benutzer, die Ausgabeempfehlungen, die zugrunde liegenden Argumente und die verwendeten Quellen besser zu verstehen. Sie erhöht die Transparenz des CDS-Systems. Für jede Entscheidungsregel könnte idealerweise in einem Entscheidungsregelprofil, so wie in einem Leitlinienaussagenprofil bei Leitlinien [22], der Originaltext einer Leitlinienempfehlung, sowie Angaben zu Originalquelle, Autoren der Empfehlung, Evidenzgrad, Empfehlungsgrad, klinischen Nutzen und Risiken aufgezeigt werden. Die Benutzeroberfläche ist der Mensch-Maschine-Kommunikationsmechanismus für das Aufnehmen von spezifischen Patientendaten, also die Eingaben durch den Benutzer, und für das Anzeigen von Ergebnissen, d. h. die Ausgaben an den Benutzer. Eine Patientendatenbank ermöglicht es CDSS-Benutzern, CDSS-spezifische Patientendaten, also die Ergebnisse des klinischen Mensch-Maschine-Entscheidungsprozesses, abzuspeichern und somit auch zu dokumentieren.

\section{Funktionsweise von CDS-Systemen}

Klinische Informationen werden in das CDSS eingegeben, indem der Benutzer aus vordefinierten Eingabedaten auf der Benutzeroberfläche eine Auswahl vornimmt. Diese Eingabedaten dienen als IF-Bedingungen der klinischen Entscheidungsregeln. Eingabedaten (Bedingungen) beschreiben vor allem die klinische Situation des Patienten und umfassen Befunde aus der Anamnese, der körperlichen Untersuchung, den Laborergebnissen und anderen klinischen Daten [1]. Die vordefinierten Eingabedatenfelder werden strukturiert (digitalisiert), maschinenlesbar angelegt, sodass der Benutzer Ja-/Nein-Entscheidungen treffen oder numerische Zahlen eingeben kann. Basierend auf der Eingabe des Benutzers (IF-Bedingungen der Entscheidungsregeln) und der automatisierten Argumentation im Hintergrund werden dem Benutzer dann eine oder mehrere digitalisierte klinische Empfehlungen (Ausgaben, DANN-Aktionen) vom CDS-System angezeigt.

\section{Transformation von Leitlinien in CDS-Tools}

Die digitale Transformation einer vollständigen narrativen Leitlinie umfasst die Übersetzung aller ausführbaren narrativen Leitlinienempfehlungen in maschinenlesbare digitale Eingabe- und Ausgabedatenelemente, die klinischen Entscheidungsregeln und die Zusammenstellung aller digitalen klinischen Entscheidungsregeln der Leitlinie (mit ihren digitalen Datenelementen) zu einem einzigen digitalen, maschinenlesbaren Artefakt, einem CDSTool.

Beim problembasierten CDS werden alle Entscheidungsregeln, die erforderlich sind, um ein bestimmtes klinisches Problem zu lösen, in ein CDS-Tool integriert. Das Tool stellt eine umfassende Problemlösungsstrategie für ein bestimmtes Patientenproblem dar. Ein problembasiertes CDS-Tool sollte dabei genau die Patientendaten vom Benutzer anfordern (oder aus anderen Quellen, z. B. der elektronischen Patientenakte), die gesammelt werden müssen, um ein leitlinienkonformes Management des Patientenproblems zu demonstrieren.

Da sich einzelne Leitlinien normalerweise auf ein (Lawrence-Weed-)Problem [16] der Problemliste beziehen, also entweder eine etablierte Diagnose (z. B. Herzinsuffizienz) oder einen ungeklärten Befund (z. B. Synkope), kann ein leitlinienbasiertes CDS-Tool auch als problembasiertes CDS-Tool angesehen werden, als eine leitlinienkonforme, digitale Problemlösungsstrategie für ein Patientenproblem. Leitlinienbasierte CDS-Tools führen Ärzte Schritt für Schritt und digital durch die Anwendung des aktuellen klinischen Standards. Nach der Abfrage der Patienteneigenschaften in einer bestimmten Reihenfolge werden dem Arzt leitlinienbasierte Empfehlungen angezeigt.

\section{Anforderungen und Möglichkeiten zukünftiger CDS-Systeme}

Trotz der durch Studien demonstrierten Nutzen kommen CDS-Systeme im klinischen Alltag aktuell nach wie vor nicht regelmäßig zum Einsatz. Im Folgenden sollen Mängel bisheriger CDS-Ansätze diskutiert werden, die in zukünftigen Systemen ggf. vermieden werden könnten. Es sollen außerdem weitere grundsätzliche Probleme der klinischen Medizin benannt werden, die durch die Anwendung von CDS-Systemen möglicherweise ebenfalls adressiert werden könnten. Es sollen Anforderungen und neue Möglichkeiten an zukünftige CDS-Systeme tabellarisch aufgezeigt werden ( $\triangleright$ Tab. 2 und $\mathbf{3}$ ). 
- Tab. 2 Anforderungen an zukünftige CDSS.

\section{Probleme bisheriger CDSS-Ansätze}

Abhängigkeit von bestehenden elektronischen Patientenakten-Systemen: CDS-Systeme wurden bisher meist direkt in elektronische Krankenhausinformationssysteme (KIS) implementiert. Jedes KIS-System zeichnet sich aber durch seine spezifische Architektur und Einschränkungen aus und erfordert spezifische Entwicklungsansätze. KIS-spezifische Arbeiten können später dann nicht auf andere Krankenhaus- und Arztpraxissysteme skaliert werden.

Fehlende Datenmodelle und Interoperabilitätsstandards: CDS-Systeme wurden bisher meist in proprietären Formaten in KIS-Systeme und Anwendungen integriert, sodass später praktisch kein Datenaustausch mit anderen Systemen stattfinden konnte.

Abhängigkeit von Softwareentwicklern: CDS-Systeme wurden bisher meist von Programmierern unter Anleitung von Ärzten eingerichtet. Änderungen, z. B. von Entscheidungsregeln, wurden von Programmierern in das CDSS integriert. Verbesserungs- und Aktualisierungsprozesse wurden dadurch dramatisch verlangsamt.

Nicht problembasierter CDS: Historische CDS-Systeme konzentrierten sich meist auf die Übermittlung von Warnungen (engl. alerts) an Ärzte, z. B. das Aufzeigen möglicher Ursachen für pathologische Laborwerte, wodurch bei jedem Anzeigen der Arbeitsablauf des Arztes unterbrochen wird.

Mangelnde Bereitstellbarkeit: Historische CDS-Systeme wurden nicht so eingerichtet, dass die Systeme einfach zu installieren waren und neu entwickelte CDS-Artefakte nachgeliefert werden können.

Mangelnde Agilität: Die Produktion von CDS-Inhalten dauert bisher viel zu lange.

Mangel an mobilen Geräten: historisch wurden CDS-Artefakte in KIS implementiert, die auf Computern oder größeren Systemen liefen.

Fehlender Plattformansatz: CDS-Systeme wurden historisch nur auf lokaler Basis zu spezifischen klinischen Problemen und für bestimmte institutionelle Umgebungen eingerichtet.

Mangel an Standardisierung klinischer Entscheidungen und Aktivitäten: Klinische Medizin umfasst so viele Teilgebiete, Krankheiten und spezifische Manifestationen, dass ärztliche Entscheidungsaufgaben als kleinteilig, „manuell“ und kaum beschreibbar gelten. Eine Standardisierung und Automatisierung der Prozesse auf lokaler Ebene ist zu zeitaufwendig, ineffizient und wird daher meist nicht durchgeführt.

Mangelnde Beteiligung medizinischer Fachgesellschaften: historisch wurde CDS meist ohne Beteiligung medizinischer Fachgesellschaften entwickelt, ohne ihre organisatorische und wissenschaftliche Kraft und ohne ihr Engagement bei der Entwicklung evidenzbasierter Leitlinien.

Fehlende Vorhersage der Ergebnisse: Ohne auf Evidenz zu beruhen, können CDS-Systeme auch keine Wahrscheinlichkeiten der zu erwartenden Outcomes angeben.

Mangel an evidenzbasierter Medizin, EbM-Leitlinien: Historisch bestand klinische Medizin in der Anwendung tradierten Wissens und klinischer Erfahrung des Arztes (Expertenwissen, Expertenmeinung). Medizin ist inzwischen zunehmend evidenzbasiert, beruht also auf Nachweisen über Wirkung und Sicherheit der Anwendungen aus klinischen Studien.

\section{Anforderungen an zukünftige CDSS}

Unabhängigkeit von KIS-Systemen: Auch wenn Benutzer (Ärzte) wünschen, „das muss gleich in meinem KIS-System funktionieren“, CDSSSoftware und CDSS-Architektur muss unabhängig sein von den bereits vorhandenen elektronischen Krankenhaus- und Arztpraxissystemen. Ansonsten ist CDS limitiert auf einen Standort und ist nicht übertragbar. (Lösung s. Datenstandards, s. u.)

Anwendung internationaler Datenstandards: CDS muss basieren auf soliden Datenmodellen, interoperablen Formaten und international anerkannten Codierungsstandards, um in jedes Computersystem integriert werden zu können, und damit z. B. doppelte Dateneingabe zu vermeiden. Diese Standards (z. B. FHIR, SNOMED, LOINC) wurden erst in den letzten Jahren entwickelt.

Unabhängigkeit von Programmierern: CDS-Systeme sollten so eingerichtet sein, dass kein Programmierer erforderlich ist, um „Inhaltsanteile“ einzurichten oder abzuändern. Es sollte ein CDSS-Editor vorhanden sein, mit dem Nichtprogrammierer, auch Endnutzer, das CDSS einrichten und modifizieren können.

Leitlinienbasierter CDS: CDS-Systeme sollten zukünftig vollständige Lösungen zu spezifischen Patientenproblemen anbieten. Alle anwendbaren Handlungsanweisungen, die erforderlich sind, um ein Patientenproblem (z. B. akute Herzinsuffizienz) ausreichend zu versorgen, sollten z. B. in je einem leitlinienbasierten CDS-Tool dargestellt werden.

CDS-Systeme sollten schnell installierbar sein (SaaS/Software-as-aService, iPaaS/Integration-Platform-as-a-Service), CDS-Tools sollten schnell lieferbar und sofort nutzbar sein.

Schnelle Aktualisierung: CDSS sollten schnell aktualisiert werden können.

CDS-Systeme sollten heute live am „Point of Care“ auf mobilen Geräten (Smartphones, Tablets) verwendet werden.

CDS-Artefakte sollten auf einer nicht lokalen, z. B. nationalen oder internationalen Ebene angefertigt und dann über das Plattformsystem an lokale Institutionen geliefert werden.

Leitlinienbasierter CDS bietet die Möglichkeit zur Standardisierung und Halbautomatisierung ärztlicher Basisaufgaben, d. h. sie überregional zu beschreiben, diese Standards an lokale Institutionen zu liefern und dann ggf. lokal angepasst anzuwenden.

Die Beteiligung wissenschaftlicher Fachgesellschaften am kompletten Wissens- und Entwicklungszyklus klinischer Leitlinien und entsprechender CDS-Tool-Artefakte, inkl. einer wissenschaftlichen Unterstützung und eines Engagements bei der Vermarktung, erscheint essenziell für den Erfolg von CDS.

Evidenzbasierte CDS-Tools können als prädiktive (Prognose-)Instrumente angewendet werden für die Vorhersage von Ergebnissen (Nutzen, Schäden) der Interventionen.

Leitlinienbasierte CDS-Systeme sind evidenzbasiert und stellen somit Evidenz dar. Zu vielen klinischen Anwendungen gibt es aber keine Studien, keine Evidenz und auch keine evidenzbasierten Leitlinien. CDSSysteme könnten in diesen Fällen evtl. auch angewendet werden, um vereinfacht Ergebnisdaten (Realweltdaten, RWD) und Realweltevidenz (RWE) zu generieren. 
- Tab. 3 Lösungsvorschläge durch CDSS.

\section{weitere aktuelle Probleme}

Mangelnde Verfügbarkeit von Leitlinien in der klinischen Versorgung und mangelnde Implementierung der neuesten klinischen Standards, Leitlinien werden nicht angewendet.

Exponentiell anwachsendes medizinisches Wissen: Ärzte können sich das aktuelle medizinische Wissen einfach nicht mehr in ausreichender Weise merken. Das Wissen ändert sich zu oft, zu schnell und ist zu umfangreich.

Mangelnde Anleitung junger Ärzte: Juniorärzte brauchen Anleitung, Aufsicht und Hilfe. Sie wollen sich auf die Patientenarbeit konzentrieren.

Es fehlt ein Qualitätsmanagementsystem in der Patientenversorgung, bei dem Prozesse im Voraus festgelegt, planmäßig durchgeführt und so dokumentiert werden, dass sie anschließend überprüft werden können.

mangelnde Transparenz (was wurde getan? warum?), mangelnder Informationstransfer zwischen Versorgungsteams, auch sektorenübergreifend

Behandlungsfehler, die zusätzliche Kosten verursachen durch Korrekturmaßnahmen, Schadensersatz, negative Kundenwahrnehmung und Erhöhung der Haftungsprämien

Zusatzkosten durch nicht indizierte harmlose, aber kostspielige Anwendungen, z. B. unnötige und Doppeluntersuchungen

zunehmende Dokumentationspflichten wegen Qualitätssicherung, Compliance, Abrechnung, Krankenversicherung und Abwehr von Schadensersatzansprüchen führen zu zusätzlichen Belastungen von Ärzten

Nichterfüllung der gesetzlichen Anforderungen: CDS-Systeme wurden bisher meist lokal als wissenschaftliche, nicht kommerzielle Studienprojekte aufgesetzt. Die regulatorischen Vorgaben durch MedizinproduktRichtlinie (MDD, 1992) bzw. Verordnung (MDR, 2017) wurden dabei letztendlich nicht erfüllt.

\section{Lösungsvorschläge durch CDSS}

Leitlinienbasierte CDS-Systeme könnten schnell geliefert werden und somit die schnelle Implementierung des neuesten klinischen Standards in die allgemeine Praxis erleichtern.

Aktuelle leitlinienbasierte CDS-Tools könnten dem Arzt stets den aktuellen klinischen Standard demonstrieren, d. h. live während des klinischen Versorgungsprozesses das evidenzbasiert indizierte Patientenmanagement anzeigen.

Mit leitlinienbasierten CDS-Tools erhalten junge Ärzte eine direkte Anleitung. Sie können sich auf ihre Patientenarbeit konzentrieren. Dadurch Generierung eines attraktiveren Arbeitsumfelds.

Implementierung eines CDS-basierten QMS, bei dem Prozesse im Voraus leitlinienbasiert geplant, dann entsprechend durchgeführt und dokumentiert werden, sodass sie später auditierbar sind.

verbesserter Informationsfluss durch CDS, da für Nachversorgende erkennbar wird, was warum getan wurde

Vermeiden von Behandlungsfehlern durch Anwendung von CDS bedeutet größere Sicherheit und geringere Zusatzkosten durch Korrekturen, Schadensersatz, negative Kundenwahrnehmung und Erhöhung der Haftungsprämien

Vermeiden von Zusatzkosten durch nicht indizierte Anwendungen

durch CDS-Dokumentation aller leitlinienkonformen Entscheidungen schon während des Prozesses der Gesundheitsversorgung Erfüllung der zunehmenden Anforderungen an Qualitätssicherung, Compliance, Abrechnung, Krankenversicherung und Abwehr von Schadensersatzansprüchen

Für einen nachhaltigen Erfolg von CDS-Systemen, d. h. für die Skalierung eines Systems und die Anwendung an unterschiedlichen Standorten ist die Erfüllung aller regulatorischen Vorgaben unabdingbar, u. a. durch Medizinprodukt-Verordnung, Datenschutz, Datensicherheit.

\section{Schlussfolgerung}

Um Qualität, Effizienz, Sicherheit, Transparenz und Outcomes (QESTO) in der klinischen Medizin weiter zu steigern, sollten leitlinienbasierte Systeme zur klinischen Entscheidungsunterstützung weiterentwickelt und angewendet werden. Dies erfordert die Zusammenarbeit aller Interessenvertreter, d. h. der benutzenden Ärzte, der Evidenz- und Leitlinienentwickler (d. h. der Fachgesellschaften), der IT-Spezialisten sowie der Vertreter von CDS-entwickelnden Institutionen.

\section{Interessenkonflikt}

Der Autor gibt an, dass kein Interessenkonflikt besteht.
Autoren

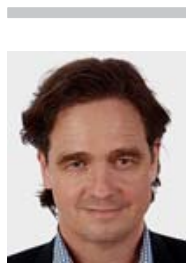

Korrespondenzadresse

\section{Dr. med. Philipp Börm}

Börm Bruckmeier Verlag GmbH

Emil-Geis-Straße 4

82031 Grünwald

Deutschland

Tel.: 089/6977810

Fax: $089 / 69778128$

philipp.boerm@media4u.com 
[1] Berner ES. Clinical Decision Support Systems, Theory and Practice. 3rd ed. New York: Springer; 2016. doi:10.1007/978-0387-38319-4

[2] Greenes RA. Clinical Decision Support, the Road to broad Adoption. 2nd ed. London: Academic Press; 2014

[3] Field M], Lohr KN. Clinical Practice Guidelines: Directions for a new Program. Institute of Medicine. Washington, DC: National Academies Press; 1990

[4] Graham R, Mancher M, Wolman DM, Greenfield S, Steinberg E, eds. Clinical Practice Guidelines we can trust. Institute of Medicine. Washington, DC: National Academies Press; 2011

[5] Barth J, Misra S, Aakre $\mathrm{K}$ et al. Why are clinical practice guidelines not followed? Clin Chem Lab Med 2016; 54: 1133-1139. doi:10.1515/cclm-2015-0871

[6] Kwok R, Dinh M, Dinh D et al. Improving adherence to asthma clinical guidelines and discharge documentation from emergency departments: Implementation of a dynamic and integrated electronic decision support system. Emerg Med Australas 2009; 21: 31-37. doi:10.1111/j.1742-6723.2008. 01149.x

[7] Keasberry J, Scott I, Sullivan C et al. Going digital: a narrative overview of the clinical and organisational impacts of eHealth technologies in hospital practice. Australian Health Review 2017; 41: 646-664. doi:10.1071/AH16233

[8] Bellodi E, Vagnoni E, Bonvento B et al. Economic and organizational impact of a clinical decision support system on laboratory test ordering. BMC Med Inform Decis Mak 2017; 17: 179. doi:10.1186/s12911-017-0574-6

[9] Calloway S, Akilo H, Bierman K. Impact of a clinical decision support system on pharmacy clinical interventions, documentation efforts, and costs. Hosp Pharm 2013; 48: 744-752. doi:10.1310/hpj4809-744

[10] McMullin S, Lonergan T, Rynearson C et al. Impact of an evidence-based computerized decision support system on primary care prescription costs. Ann Fam Med 2004; 2: 494498. doi:10.1370/afm.233

[11] Algaze CA, Wood M, Pageler NM et al. Use of a checklist and clinical decision support tool reduces laboratory use and improves cost. Pediatrics 2016; 137: e20143019. doi:10.1542/ peds.2014-3019

[12] O'Sullivan D, O'Mahony D, O'Conner M et al. Prevention of adverse drug reactions in hospitalised older patients using a software-supported structured pharmacist intervention: a cluster randomised controlled trial. Drugs Aging 2016; 33: 63-73. doi:10.1007/s40266-015-0329-y
[13] Chow A, Lye D, Arah O. Mortality benefits of antibiotic computerised decision support system: modifying effects of age. Sci Rep 2015; 5: 17346. doi:10.1038/srep17346

[14] Jia P, Zhang L, Chen J et al. The effects of clinical decision support systems on medication safety: an overview. PLoS One 2016; 11: 1-17. doi:10.1371/journal.pone.0167683

[15] Vermeulen K, Van Doormaal J, Zaal R et al. Cost-effectiveness of an electronic medication ordering system (CPOE/CDSS) in hospitalized patients. Int J Med Inform 2014; 83: 572-580. doi:10.1016/j.ijmedinf.2014.05.003

[16] Weed LL. Medical records that guide and teach. N Engl J Med 1968; 278: 593-600. doi:10.1056/NEJM196803142781105

[17] Weed L. Larry Weed's 1971 Internal Medicine Grand Rounds (1971). Im Internet (Stand: 30.09.2020): https://youtu.be/ qMsPXSMTpFI

[18] Walsh S. The Problem Oriented Medical Record (July 2011). Im Internet (Stand: 30.09.2020): https://www.youtube.com/ watch? $v=k 2 L W X X S 3 e u 0$

[19] Boxwala A, Rocha B, Maviglia S et al. A multi-layered framework for disseminating knowledge for computer-based decision support. J Am Med Inform Assoc 2011; 18: 132-139. doi:10.1136/amiajnl-2011-000334

[20] Centers for Disease Control and Prevention (CDC). Adapting Clinical Guidelines for the Digital Age Meeting, Connect, Collaborate, Understand. Meeting Summary (May 2018). Im Internet (Stand: 30.09.2020): https://www.cdc.gov/ddphss/ clinical-guidelines/February-2018-Meeting-Summary.html

[21] Davey J, Turner RM, Clarke M] et al. Characteristics of metaanalyses and their component studies in the Cochrane Database of Systematic Reviews: a cross-sectional, descriptive analysis. BMC Med Res Methodol 2011; 11: 160. doi:10.1186/ 1471-2288-11-160

[22] Rosenfeld R, Shiffman R, Robertson P. Clinical practice guideline development manual, third edition: a quality-driven approach for translating evidence into action. Otolaryngol Head Neck Surg 2013; 148 (1 Suppl.): S1-S55. doi:10.1177/ 0194599812467004

Bibliografie

Online publiziert 30.03.2021

OP-JOURNAL 2021; 37: 28-35

DOI 10.1055/a-1284-3193

ISSN $\quad 0178-1715$

(c) 2021. The Author(s). This is an open access article published by Thieme under the terms of the Creative Commons Attribution-NonDerivative-NonCommercial-License, permitting copying and reproduction so long as the original work is given appropriate credit. Contents may not be used for commercial purposes, or adapted, remixed, transformed or built upon. (https://creativecommons.org/licenses/by-nc-nd/4.0/)

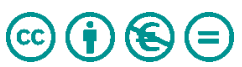

